\title{
Detecting Cholesterol Presence with Iris Recognition Algorithm
}

\author{
Ridza Azri Ramlee, Khairul Azha and Ranjit Singh Sarban Singh \\ Universiti Teknikal Malaysia Melaka (UTeM), \\ Malaysia
}

\section{Introduction}

Iris is a pigmented, round, contractile membrane of the eye, suspended between the cornea and lens and perforated by the pupil (Fig. 1). It regulates the amount of light entering the eye (Online Dictionary). According to (David J. Pesek, 2010), the eyes are connected and continuous with the brain's Dura mater through the fibrous sheath of the optic nerves, and they are connected directly with the sympathetic nervous system and spinal cord. The optic tract extends to the thalamus area of the brain. This creates a close association with the hypothalamus, pituitary and pineal glands. These endocrine glands, within the brain, are major control and processing centers for the entire body. Because of this anatomy and physiology, the eyes are in direct contact with the biochemical, hormonal, structural and metabolic processes of the body. This information is recorded in the various structures of the eye, i.e. iris, retina, sclera, cornea, pupil and conjunctiva. Thus, it can be said that the eyes are a reflex or window into the bioenergetics of the physical body and a person's feelings and thoughts (David J. Pesek., 2010). There are a lot of arguments between iridologists (iridology's practitioner) and the medical's practitioner. Due to this argument, numerous studies done by the medical's practitioner found that the diagnosis done by the iridologist upon the patient is not accurate (Allie Simon et al, 1979). However the study on relationship diseases to iris changes, still continuing for example the studied done on Ocular complication of adult rheumatoid arthritis don by S.CReddy and U.R.K.Rao in 1996 found that the mean duration of the arthritis and the mean duration of seropositivity were found to be significantly higher in patients with ocular (pigmented organ in eye) complication (S.CReddy et al, 1996). Another study done on bilateral retinal detachment in acute myeloid Leukemia by (K Pavithran et al., 2003), found that ocular manifestations are common in patient with acute Leukemia. This can result from direction infiltration by neoplastic cells of ocular tissues, including optic nerve, choroid, retina, iris and ciliary body, or secondary to hematology abnormalities such as anemia, thrombocytopenia, or hyperviscosity states or retinal destruction by opportunistic infection (K Pavithran et al., 2003). The history of Iridology study on iris was done by the physician Philippus Meyens in 1670 in a book explaining that the features of the irid called Chromatica Medica. In that book he wrote that the eye (iris) contains valuable information about the body. In 1881 a Hungrarian physician, Dr. Ignatz Peczley who is claimed as the founder of modern Iridology wrote a book "Discoveries in the Field of Natural Science and Medicine, a guide to the study and 
diagnosis from the eye." He introduced the first chart of the iris explaining zone in the iris. The idea of his study on iris, begun when he was a child, he was accidentally found the Owl with broken leg. He found a dark scar in the Owl's iris that scar turned white as the leg healed (Sandy Carter, 1999). The objective of this chapter is to explain how the presence of cholesterol in blood vessel can be detected by using iris recognition algorithm. This method used the John Daugman's and Libor masek's iris recognition methods and extends the study of eyes pattern to other application and in this case, the alternative medicine that is iridology. Based on the iris recognition methods and iridology chart, a MATLAB program has been created to detect the present of cholesterol in our body. However, further analysis must be done in order to know the exact range or level of cholesterol in blood vessel.

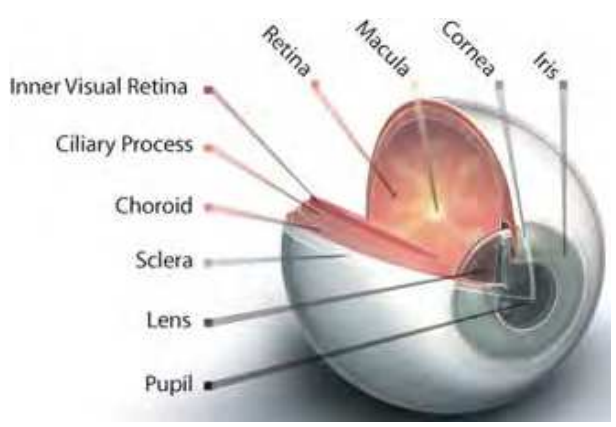

Fig. 1. Human Eye Anatomy.

\subsection{Relationship cholesterol presence with arcus senilis}

Hypercholesterolemia or a high level of cholesterol in the blood poses a significant threat to person's health. Even though it is not considered as a disease, it can be secondary to a disease and can help contribute to other many forms of diseases most notable are cardiovascular diseases. So, it is important to have our blood cholesterol levels checked. A contemporary technique to measure the cholesterol level is by doing blood test and the test is known as lipoprotein profile. The lipoprotein profile is done and preferably after a 9- to 12- hour fast and it measures the levels of total cholesterol.

The lipoprotein profile can be considered as intrusive means if it is just used merely for cholesterol screening. (N.Haq, M.D.Fox, 1991) introduced laser based technology as nonintrusive technique to measure blood cholesterol through skin. They proposed infrared (IR) absorption spectroscopic as the characterization of cholesterol in the skin. Based on U.S Food and Drug Administration (FDA), (FDA, 2004), skin contains approximately 11 percent by weight of all body cholesterol and when severe coronary artery disease is present, the numeric values obtained with the skin cholesterol test increases. Thus, the palm test for skin cholesterol is not to be useful in identifying people with less severe coronary artery disease and it is not intended to be used as a screening tool to determine the risk for coronary artery disease in general population.

In order to have a simple and non-intrusive means to be as a screening tool to detect cholesterol, we have considered alternative medicines. Iridology is one of the alternative medicines, which claims that iris pattern could reflect one's health and reveal the state of individual organs. According to iridology, cholesterol in body can be detected if there is a 
"sodium ring" in the patient's eyes. However, since there were statements that regard iridology as medical fraudulent (L.Berggren, 1985), we were looking at other medical statements that can relate cholesterol and other organs. We found out that high cholesterol can be detected from changes in iris pattern and they are called Arcus Lipoides (Arcus Senilis or Arcus Juvenilis). "Arcus senilis is a greyish or whitish arc or circle visible around the peripheral part of the corner in older adults. Arcus senilis is caused by lipid deposits in the deep layer of the peripheral cornea and not necessarily associated with high blood cholesterol. However, similar discoloration in the eyes of younger adults (arcus juvenilis) is often associated with high blood cholesterol (K.Hughes et. al., 1992)." This statement proves that iris pattern can be analyzed and used as another technique to detect cholesterol presence in body.

(Harold z. Pomerantz, 1962), conclude in his study the presence of Arcus Senilis before the age of 56 and large wrist size were found to appear with a frequency in coronary group which made their presence statistically significant at level 5\%. Hypercholesterolemia was common finding in coronary patient who demonstrated Arcus Senilis and greying of hair. According to (Jae-Young Um et. al, 2005) although iridology has been criticized as an unfounded diagnostic tool, many iridologists are presently practicing in many areas. In Germany, $80 \%$ of Heilpraktiker (non-medically qualified health practitioners) practice iridology (Ernst, 2000). In this study, (Jae-Young Um et. al, 2005) investigated the ACE genotypes of hypertensive patients classified by their iris constitutions. As a result, $74.7 \%$ of hypertensive patients were neurogenic or cardio-renal connective tissue weakness type. Also, the frequencies of DD genotype were significantly higher in hypertensive patients than in controls. These results are consistent with the reports that DD genotype was associated with hypertension (Staessen et al., 2001). Therefore, (Jae-Young Um et. al, 2005) present the results support that $\mathrm{D}$ allele is a candidate gene for hypertension, and suggest an apparent relationship between ACE genotype and iris constitutions, as well as the novel possibility of molecular genetics understanding of iridology.

\section{Eye image}

The eye is the organ of sight, a nearly spherical hollow globe filled with fluids (humors). The outer layer or tunic (sclera, or white, and cornea) is fibrous and protective. The middle tunic layer (choroid, ciliary body and the iris) is vascular. The innermost layer (the retina) is nervous or sensory. The fluids in the eye are divided by the lens into the vitreous humor (behind the lens) and the aqueous humor (in front of the lens). The lens itself is flexible and suspended by ligaments which allow it to change shape to focus light on the retina, which is composed of sensory neurons (NLM, 2010). Fig. 1 shows the anatomy of human eye which contain the area of sclera and iris for references. The iris image needs to be extract from the original eye image. This solid iris image will be used in this system to verify the presence of cholesterol. Thus it is vital to isolate this part (iris) from the whole unwanted part in the eye (sample). This separation or segmentation is the process of remove the outer part of the eye (outside the iris circle), in order to get solid image of iris that useful for localisation the cholesterol lipid. Generally this eye breaks up into two parts, the first part is the inner region which is the iris and pupil boundary and the second part is the outer regions, the iris and sclera boundary. The quality of the images is very important to get the best result, thus the images should not have any impurities that can cause miss localization. These impurities include the flash reflection from camera and wrong angle of image capture. 
In this project the sample of eye is very vital because analysis base on the data from human eyes. The easier way to have these samples is by using free database source. These database are freely available iris images database, they give permission to all researcher, student and et cetera for research or educational purpose. There are a few free database sources that can found in website, these databases such as CASIA, MMU, UPOL, UBIRIS en cetera. To use these database someone need to write email or send the e-form to the authors and state the particular information such as name, status, organization and purpose to use database. Some of these databases have the restriction such as username and password, and these data will be given base on request by the users when they email the author for use their database.

\subsection{UBIRIS}

UBIRIS database is comprised of 1877 images collected from 241 subjects within the University of Beira Interior 6 in two distinct sessions and constituted, at its release date, the world's largest public and free iris database for biometric purposes.

\subsection{CASIA}

(CASIA, 2003), iris image database (version 1.0, the only one that we had access to) includes 756 iris images from 108 eyes, hence 108 classes. For each eye, 7 images are captured in two sessions, where three samples are collected in the first and four in the second session. Similarly to the above described database, its images were captured within a highly constrained capturing environment, which conditioned the characteristics of the resultant images. They present very close and homogeneous characteristics and their noise factors are exclusively related with iris obstructions by eyelids and eyelashes (Fig. 2). Moreover, the post process of the images filled the pupil regions with black pixels, which some authors used to facilitate the segmentation task.
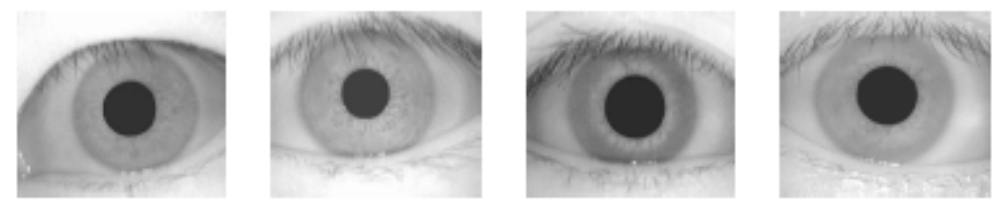

Fig. 2. Examples of iris images from the CASIA database.

\subsection{MMU}

The Multimedia University has developed a small data set of 450 iris images (MMU). They were captured through one of the most common iris recognition cameras presently functioning (LG IrisAccessR 2200). This is a semi-automated camera that operates at the range of 7-25 cm. Further, a new data set (MMU2) comprised of 995 iris images has been released and another common iris recognition camera (Panasonic BM-ET100US Authenticam) was used. The iris images are from 100 volunteers with different ages and nationalities. They come from Asia, Middle East, Africa and Europe and each of them contributed with five iris images from each eye. Obviously, the images are highly homogeneous and their noise factors are exclusively related with small iris obstructions by eyelids and eyelashes (Fig. 3). 

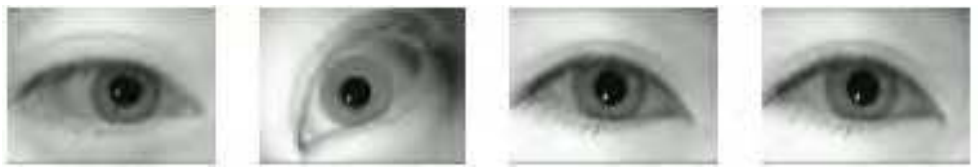

Fig. 3. Examples of iris images from the MMU database.

\subsection{Medical website}

For this project the limitation to achieve the subject or patient to get real image of eye sample, the best place to get these real eye images is at ophthalmology department since this department deal with various case of eye problem that later can be refer to Arcus Senilis problem. For this reason, the samples only can be used from free source as from medical. The medical iris images can be obtain from TedMontgomery, National Library of Medicine, Mediscan clipart library licensed medical pictures and Mayo clinic and foundation for medical education and research medical and research training Fig. 4. show a few sample from above website.
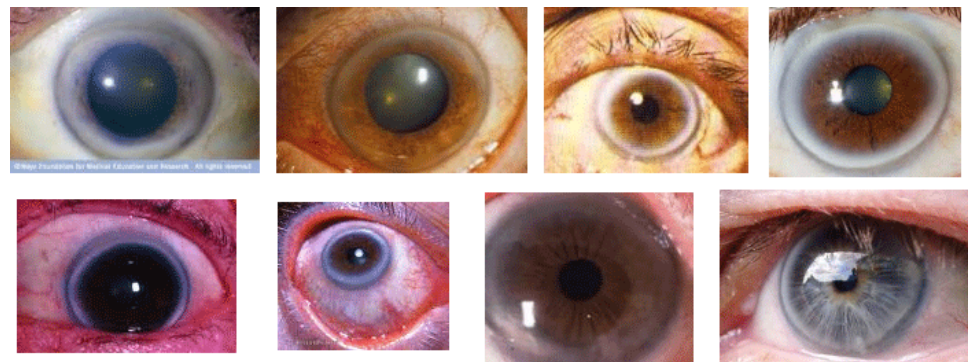

Fig. 4. Examples of iris images from medical website.

\section{Iris recognition}

Iris recognition is one of the most widely implemented biometric systems in use today. John Daugman is said to have developed the most widely used algorithms and most efficient methods of recognition, but there have been many new findings and algorithms (J. Daugman, 2004). (L. Masek, 2003) has verified the uniqueness of human iris patterns and developed "an open source" iris recognition system.

\subsection{Hough transform}

The Hough transform is a standard computer vision algorithm that can be used to determine the parameters of simple geometric objects, such as lines and circles, present in an image. The circular Hough transform can be employed to deduce the radius and centre coordinates of the pupil and iris regions. An automatic segmentation algorithm based on the circular Hough transform is employed (Theodore \& Richard.W 2002). Firstly, an edge map is generated by calculating the first derivatives of intensity values in an eye image and then set the threshold base on the result. From the edge map, votes are cast in Hough space for the parameters of circles passing through each edge point. These parameters are the centre coordinates $\mathrm{x}_{\mathrm{c}}$ and $\mathrm{y}_{\mathrm{c}}$, and the radius $\mathrm{r}$, which are able to define any circle according to the equation 


$$
x_{c}^{2}+y_{c}^{2}+r^{2}=0
$$

A maximum point in the Hough space will correspond to the radius and centre coordinates of the circle best defined by the edge points. (Theodore \& Richard.W 2002) and Kong and Zhang also make use of the parabolic Hough transform to detect the eyelids, approximating the upper and lower eyelids with parabolic arcs, which are represented as;

$$
\left(\left(-x-h_{j}\right) \sin \theta_{j}+\left(y-k_{j}\right) \cos \theta_{j}\right)^{2}=a_{j}\left(\left(x-h_{j}\right) \cos \theta_{j}+\left(y-k_{j}\right) \sin \theta_{j}\right)
$$

$a_{j}$ controls the curvature, $\left(h_{j}, k_{j}\right)$ is the peak of the parabola and $\theta_{j}$ is the angle of rotation relative to the $\mathrm{x}$-axis.

In performing the preceding edge detection step, (Theodore \& Richard.W 2002) bias the derivatives in the horizontal direction for detecting the eyelids, and in the vertical direction for detecting the outer circular boundary of the iris. The motivation for this is that the eyelids are usually horizontally aligned, and also the eyelid edge map will corrupt the circular iris boundary edge map if using all gradient data. Taking only the vertical gradients for locating the iris boundary will reduce influence of the eyelids when performing circular Hough transform, and not all of the edge pixels defining the circle are required for successful localisation. Not only does this make circle localisation more accurate, it also makes it more efficient, since there are less edge points to cast votes in the Hough space.

\subsection{Segmentation}

This segmentation (localization) process is to search for the centre coordinates of the pupil and the iris along with their radius. These coordinates are marked as $c_{i}, c_{p}$, where $c_{i}$ represented as the parameters of $\left[x_{c}, y_{c}, r\right]$ of the limbic and iris boundary and $c p$ represented as the parameters of $\left[x_{c}, y_{c}, r\right]$ of the pupil boundary. It makes use of (Theodore \& RichardWildes 2002) method to select the possible centre coordinates first. The method consist of threshold followed by checking if the selected points (by threshold) correspond to a local minimum in their immediate neighborhood these points serve as the possible centre coordinates for the iris. Once the iris has been detected (using Daugman's method), the pupil's centre coordinates are found by searching a 10x10 neighbourhood around the iris centre and varying the radius until a maximum is found (J. Daugman, 2004). The input for this function is the image to be segmented and the input parameters in this function including rmin and rmax (the minimum and maximum values of the iris radius). The range of radius values to search for was set manually, depending on the database used. For the CASIA database (example, iris3.bmp), rmin is set to 55 pixels and rmax is set to160 pixel.

The input for this function is the image to be segmented and the input parameters in this function including rmin and rmax (the minimum and maximum values of the iris radius).The range of radius values to search for was set manually, depending on the database used. For the CASIA database (example, iris3.bmp), rmin is set to 55 pixels and rmax is set to 160 pixels. The sample of Arcus Senilis eye (arcus7.bmp) is downloading from (Mediscan, 2000), for this image the rmin is set to 70 pixels and rmix is set to 260 pixels. Table 1 shows the others sample run for segmentation process. 


\begin{tabular}{|c|r|r|}
\hline Image & rmin & rmax \\
\hline Iris1.bmp (CASIA) & 75 & 260 \\
\hline Iris2.bmp (CASIA) & 75 & 260 \\
\hline Iris3.bmp (CASIA) & 75 & 260 \\
\hline Iris4.bmp (CASIA) & 75 & 260 \\
\hline Iris6.bmp (CASIA) & 75 & 260 \\
\hline c_mo1.bmp & 50 & 83 \\
\hline c_mo2.bmp & 75 & 260 \\
\hline normal1.bmp & 75 & 260 \\
\hline Arcus1.bmp (Medical Web) & 80 & 260 \\
\hline Arcus2.bmp (Medical Web) & 80 & 260 \\
\hline Arcus3.bmp (Medical Web) & 80 & 260 \\
\hline Arcus4.bmp (Medical Web) & 80 & 260 \\
\hline Arcus5.bmp (Medical Web) & 80 & 260 \\
\hline Arcus6.bmp (Medical Web) & 80 & 260 \\
\hline Arcus7.bmp (Medical Web) & 80 & 260 \\
\hline Arcus2.bmp (Medical Web) & 80 & 260 \\
\hline Arcus2.bmp (Medical Web) & 80 & 260 \\
\hline Arcus2.bmp (Medical Web) & 80 & 260 \\
\hline ubiris1.bmp (UBIRIS) & 85 & 260 \\
\hline ubiris2.bmp (UBIRIS) & 85 & 260 \\
\hline ubiris2.bmp (UBIRIS) & 85 & 260 \\
\hline
\end{tabular}

Table 1. Shows the example of the images and their rmin and rmax values.

The output of this function will be the value of $c_{p}$ and $c_{i}$, which is the value of $\left[x_{c}, y_{c}, r\right]$ for the pupilary boundary and the limbic / iris boundary and also the segmented image.
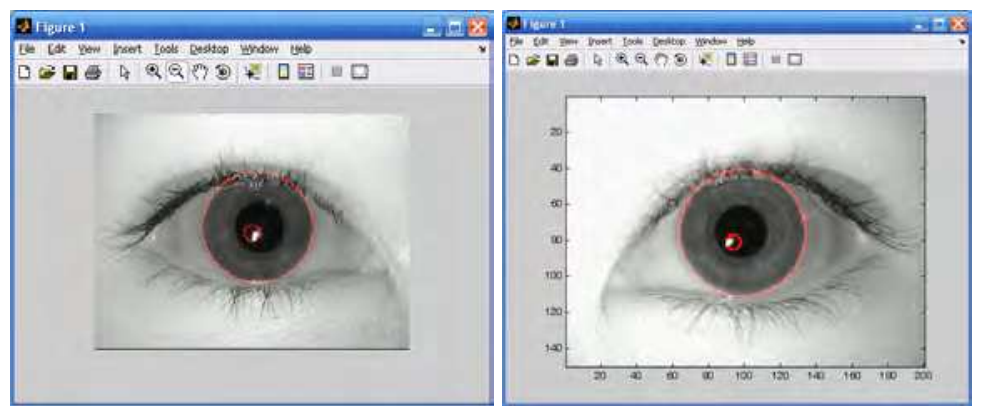

Fig. 5. An example where segmentation fails. The segmentation is failing to detection correctly the edges of the pupil border, but it segmented illumination light.

The program has be run on the image from CASIA as in the Fig. 5, this image give wrong detection on pupil boundary because segmentation on pupil is segmented on the illumination light rather than segmented the pupil boundary. Another example as shows in the Fig. 6, also fail to determine the edge of pupil but it detect the edge of the impurity illumination light. This will effect to the quality of the segmentation eyes image, cause to 
imperfectly to detect iris and pupil boundary region of the eyes. But luckily the significant area of white ring (Arcus Senilis) lay at the boundary of sclera or iris up to the pupil, so as long as the segmentation done correctly on the iris it can considered succeed. This segmentation image will be crop base on the value of iris's radius.

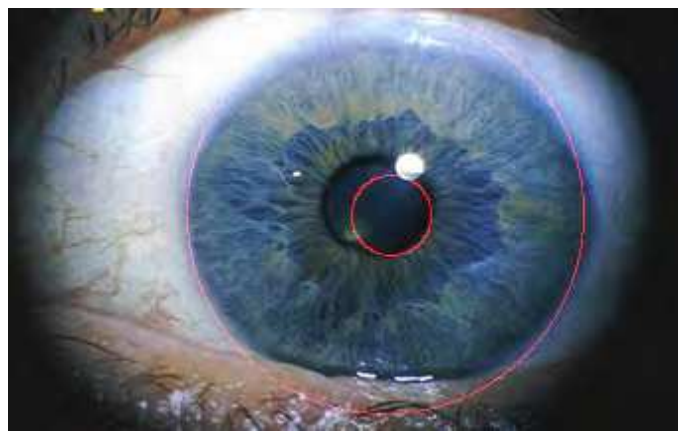

Fig. 6. Another example where segmentation fails. Canny edge detection fails to find the edges of the pupil border, but it takes edge of impurity of the illumination light.

The problem with this image is the existing of illumination from camera (on top of the pupil) will cause miss detection of iris and pupil boundary. This process consider failure if the purpose of this segmentation process is used for biometric iris recognition, but for this system since the image only need to be analyze in 30 percent from the limbic (border of iris) so this result can be used.

\subsection{Normalization}

After the iris is localized the next step is normalization (iris enrolment). It is a process after localization (segmentation) is to change the iris region to the fixed Fig. in order to make further analysis. From the process of normalization, the segmented image of the eye will give the value radius pupil and the iris. This image will be crop base on the value of iris radius, so that the unwanted area will be removing (eg sclera and limbic). Therefore only the intended area can be analyzed. According to (Frank L. Urbano, 2001), Arcus Senilis, or Corneal Arcus, is described as a yellowish-white ring around the cornea that is separated from the limbus by a clear zone 0.3 to $1 \mathrm{~mm}$ in width. It is caused by extracellular lipid deposition in the peripheral cornea, with the deposits consisting of cholesterol, cholesterol esters, phospholipids, and triglycerides. The fatty acids that make up many of the deposited lipid molecules include palmitic, stearic, oleic, and linoleic acids. Normally the area of white ring (Arcus Senilis), occurs from the sclera/iris up to 20 to 30 percents toward to pupil, so this is the only the main area that have to be analyzed. The other reason to normalize is to make the analysis process become easier rather than to examine the eye in circular shape. In rectangular shape analyze can be done either from top to bottom or from bottom to top.

(J. Daugman, 2004) describes details on algorithms used in iris recognition. He has introduced the Rubber Sheet Model that transforms the eye from circular shape into rectangular form and it is shown in Fig.7. This model remaps all point within the iris region to a pair of polar coordinates $(r, \theta)$, where $\theta$ is the angle $[0,2 \pi]$ and $r$ is on the interval $[0,1]$. 


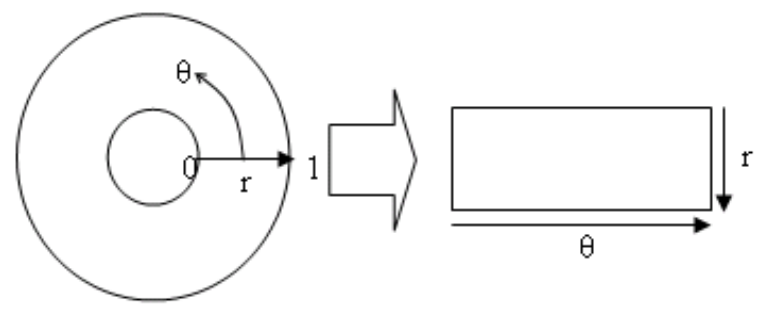

Fig. 7. Daugman's rubber sheet model.

The remapping of the iris image $\mathrm{I}(\mathrm{x}, \mathrm{y})$ from Cartesian coordinates to the normalized nonconcentric polar representation can be modelled as:

$$
I(x(r, \theta), y(r, \theta)) \rightarrow I(r, \theta)
$$

With

$$
\begin{gathered}
x(r, \theta)=(1-r) x_{p}(\theta)+r x_{l}(\theta) \\
y(r, \theta)=(1-r) y_{p}(\theta)+r y_{l}(\theta)
\end{gathered}
$$

Where $I(x, y)$ is the iris region image, $(x, y)$ are the original Cartesian coordinates, $(r, \theta)$ are the corresponding normalised polar coordinates, and $x_{p}, y_{p}$ and $x_{l}, y_{l}$ are the coordinates of the pupil and iris boundaries along the $\theta$ direction. The localization of the iris and the coordinate system is able to achieve invariance to $2 \mathrm{D}$ position and size of the iris, and to the dilation of the pupil within the iris.

The normalization process can be illustrated in Fig. 8. It is done by taking the reference point from the centre of the pupil and radial vectors pass via the iris area. There are two important data points along each radial line which are radial resolution for radial line in pupil and angular resolution for radial line around the iris region. Since the pupil can be non-matching with the iris therefore it need to remap to rescale the points depending to the angle around the iris and pupil. This formula given by:

$$
r^{\prime}=\sqrt{\alpha} \beta \pm \sqrt{\alpha \beta^{2}-\alpha-r_{I}^{2}}
$$

With

$$
\begin{gathered}
\alpha=o_{x}^{2}+o_{y}^{2} \\
\beta=\cos \left(\pi-\arctan \left(\frac{o_{y}}{o_{x}}\right)-\theta\right)
\end{gathered}
$$

The shift of centre of the pupil relative to the iris centre, this given by $O_{x}, O_{y}$, and $\mathrm{r}^{\prime}$ is the distance between edge of pupil and edge of the iris at the angle $\theta$ around the region, and $r^{\prime}$ is the radius of the iris. The remapping formula first gives the radius of the iris region 'doughnut' as a function of the angle $\theta$. 
A constant number of points are chosen along each radial line, so that a constant number of radial data points are taken, irrespective of how narrow or wide the radius is at a particular angle. The normalised pattern was created by backtracking to find the Cartesian coordinates of data points from the radial and angular position in the normalised pattern. From the 'doughnut' iris region, normalisation produces a 2D array with horizontal dimensions of angular resolution and vertical dimensions of radial resolution.

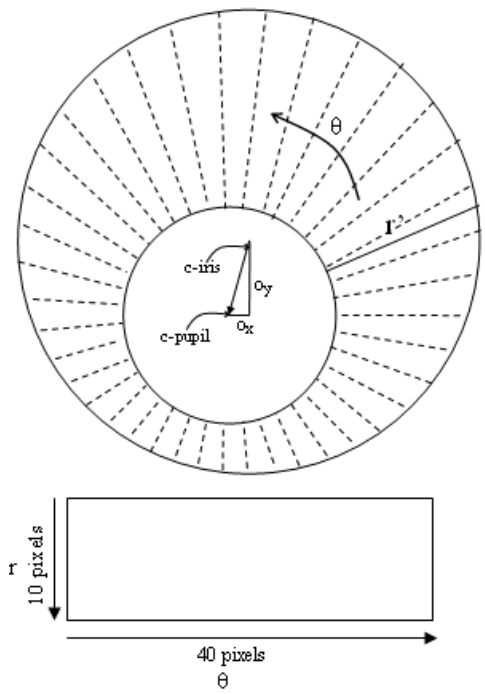

Fig. 8. Outline of the normalisation process with radial resolution of 10 pixels, and angular resolution of 40 pixels. Pupil displacement relative to the iris centre is exaggerated for illustration purposes.

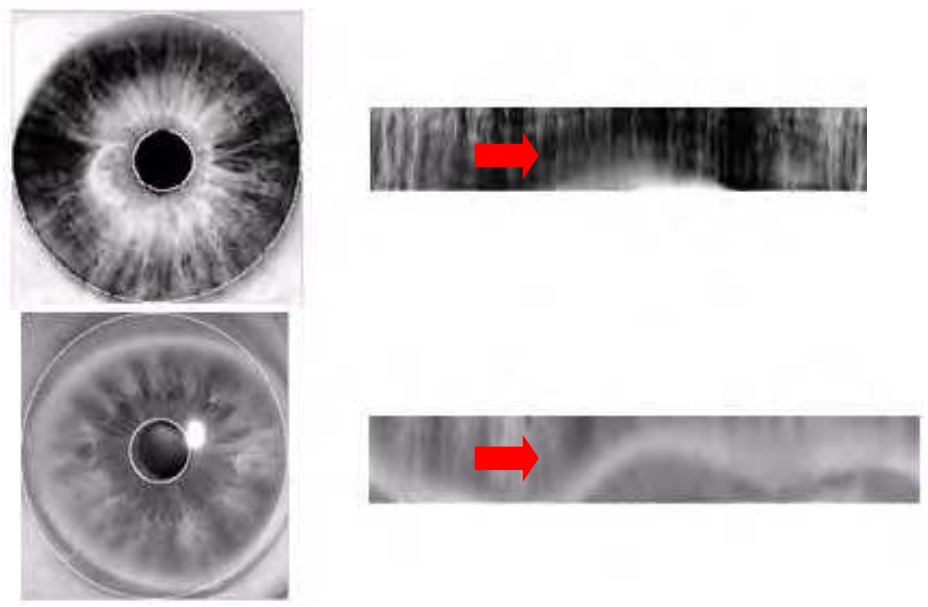

Fig. 9. Illustration of the normalization process for two images of the same iris taken under varying conditions. Top image normal eye, bottom image suspected illness eye. 
The normalisation process proved to be successful and some results are shown in Fig 9. This normalization process will transforms the segmented eye from the circular form to the rectangular shape. However normalisation output will be cropped until 30 percents, from the bottom eye (sclera and iris boundary) toward pupil.

Normalisation of two eye images of the same iris is shown in Fig 9. The pupil is smaller in the bottom image, however the normalisation process is able to rescale the iris region so that it has constant dimension. Note that rotational inconsistencies have not been accounted for by the normalisation process, and the two normalised patterns are slightly misaligned in the horizontal (angular) direction. Rotational inconsistencies will be accounted for in the matching stage.

It is difficult to do analysis if the image is in the original form therefore the image needs to be wrapped to transform the nature from circle to rectangular shape. This process only can be achieved by doing the conversion polar to rectangular.

The normalisation process proved to be successful and some results are shown in Fig. 9. However, the normalisation process was not able to perfectly reconstruct the same pattern from images with varying amounts of pupil dilation, since deformation of the iris results in small changes of its surface patterns.
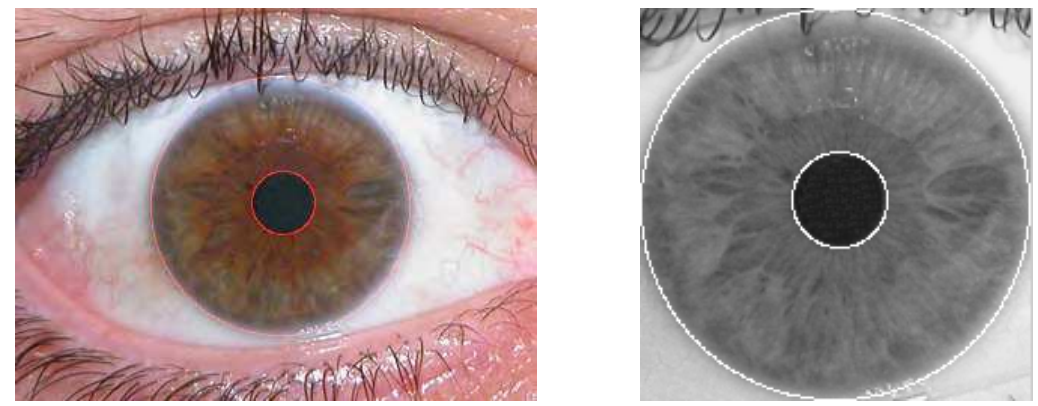

Fig. 10. Stages of localization with eye image 'ubiris1.bmp (340x260)' from the UBIRIS database left) original color eye image localization right) black and white (in gray) eye image localization.
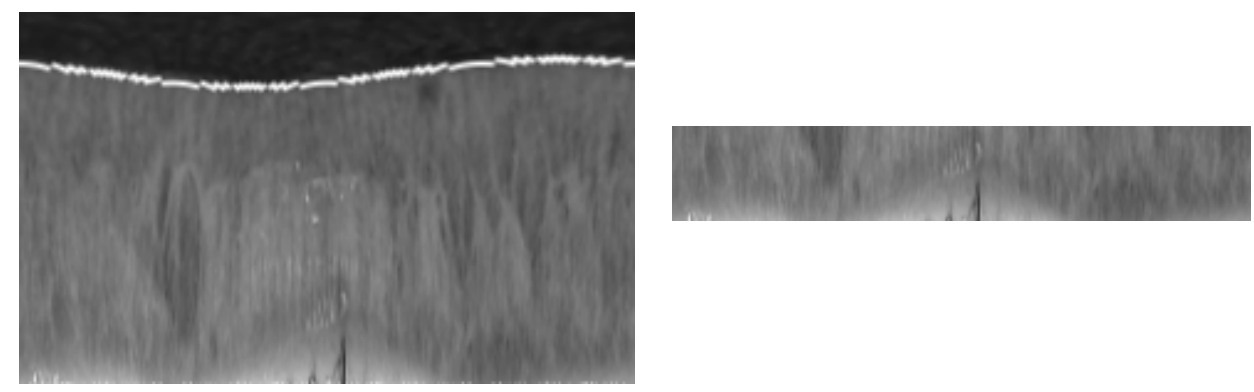

Fig. 11. Stages of normalization with eye image 'ubiris1.bmp (340x260)' from the UBIRIS database left) 100 percents display from polar to rectangular of localization eye. (Right) 30 percents display from polar to rectangular of localization eye, after crop process. 
The normalization process is used for converting the circular iris into rectangular form with fixed dimension as shown in Fig 12. We can see clearly the circle shape (Localisation) turn to rectangular shape (normalisation) and also the signs in both pictures are labelled to illustrate the upper eyelid, pupil and white dot exit during segmentation and after normalisation.

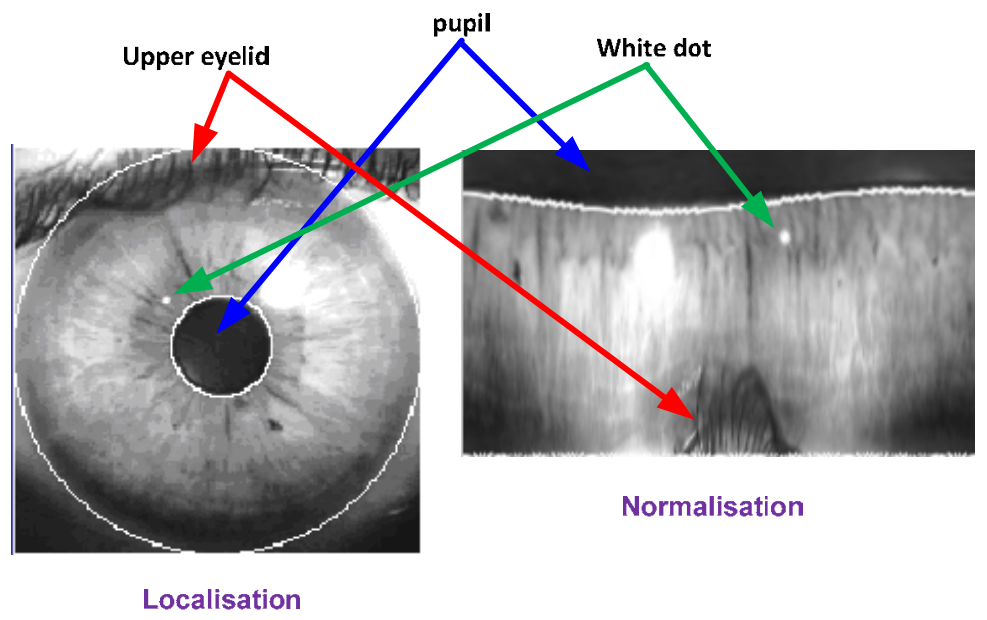

Fig. 12. Stages of normalization showing the same part in both shape circular and rectangular in the eye.

\section{Cholesterol detection system}

The Daugman's Rubber Model is the main process in the cholesterol detection system proposed in this paper. The process starts with obtaining number of normal eyes images and images of eyes with Arcus Lipids. The normal eyes are available from UBIRIS databases while the Arcus Lipids eyes are taken from iridology clinic.

The next step is to isolate the actual iris region in digital eye image. The isolation process needs to be done to segment the outer boundary for the iris and the inner boundary for the pupil. This can only be done by searching the centre point of the pupil given by $x$ and $y$ axis. Hough transform is used to detect edge of the iris and pupil circle.

Next, the image has to be analyzed and this can only be done if it is transformed to normalized polar coordinates using Rubber Model. Since the "sodium ring", terminology given in iridology, or Arcus Lipids for the greyish or whitish arc in iris is only available at the bottom of this coordinate, thus only $30 \%$ of the iris part is considered in the normalization.

Lastly, to determine whether the eye has the ring, histogram of the image has to be plotted so that the decidability can be determined using OTSU's method. The algorithm assumes the image contains two classes of pixels (e.g. foreground and background) and finds the optimum threshold separating the two classes so that their combined spread (within-class variance) is minimal. 


\section{Results}

Fig 13 shows the whole process of the cholesterol detection system using iris recognition and image processing algorithm comprises the following actions:

- $\quad$ Eye images acquire from database (CASIA, UBIRIS, MMU and medical web) or from digital camera.

- Process of pupil and iris localization and segmentation, to classify the required region.

- Attain normalization iris from circular shape to rectangular shape with full image $(100 \%)$

- $\quad$ Crop the normalization iris to $30 \%$ from full image.

- Run the normalization iris to get the histogram value.

- Using OTSU to calculate the optimum threshold to detect Arcus Lipids (Cholesterol presence).

- Results "Sodium ring detected" or "not detected" will be display in MATLAB window. This experiment used the eye images from free available database sources that can be downloaded by permission of the author of the database. The eye images also can be taken from high quality digital camera where the subject should be categorised to two groups of people; the healthy people and the suspected people with cholesterol symptom. These databases are free and can be used for research and educational purpose. Amongst these databases such as Chinese Academy of science (CASIA), Multimedia University (MMU), UBIRIS, and UPOL. And for medical images it can be used from TedMontgomery, National Library of Medicine, Mediscan clipart library licensed medical pictures and Mayo clinic and foundation for medical education and research medical and research training. For these database the eye images can be categories to two groups, first group provide grey colour images such as from CASIA and MMU database, while the second group such UBIRIS and almost all the medical website provide colour images. The reason to use eye image from these databases because these images are easy to be achieved without need to find eye images from any subject (patients).

The next process is localization and segmentation of pupil and iris this is to classify the required region. In this process two important regions need to be segmented which are pupil (the inner circular, normally black circular) and iris (the outer part, pattern and colour area). The unused part such as sclera (the white part outside the iris) will be removed because this region is not aimed for this experiment.

Eye image crop, base on iris radius; after segmentation process (the image is converted to grey scale), the next process is to cropped the eye image base on the value of iris radius. This iris radius value is obtained from the process of segmentation. In the segmentation process three important parameters are achieved, such as circular pupil parameters $(x, y$, and $r)$ for pupil and circular iris $(x, y$ and $r)$ for iris. To crop this grey scale eye image the $x, y$ and $r$ value from the iris's radius are needed because this is the outer and the biggest circular in this eye image. The result from this process will be obviously eye image contain only iris and pupil image, that can be used for next process of normalization process.

Normalization process; this process involve transforming the shape of image achieve from segmentation process (cropped eye image), where the segmentation eye image in circular shape will be change to rectangular shape, as illustrate in Fig 8 . The reason to change this shape is to make the analysis can be carried out easily compare to original shape (circular shape). If the analysis is performed in circular shape the analysis must be done around the 
circle of the eye and this will be so difficult, while analysis upon rectangular shape is more practical and easy to be done. This method transforming to rectangular shape, is been introduced by Professor John Daughman in his paper under Rubber's shape model, which this method analysis is performed upon the rectangular eye image from the bottom image up to the top of the image or vice versa. This normalized process will be transformed all area of circular shape of the eye image to become rectangular shape and the process will be mark as 100 percent normalization process output. According to Frank L. Urbano, in his medical journal (Hospital Physician Past Article 2001), the sign of cholesterol deposits can be seen 30 percents from bottom (border of sclera) of the eye image. As referring to this statement the 100 percents normalization eye image will be crop to until 30 percents remaining. This process still normalization image, where the cropping taken the part from the pupil to a middle part of iris up to 70 percents area involve. The reason of this process because the white ring (cholesterol's deposit) usually affect in this area which is 30 percents from the bottom of sclera or iris region up to the top of the pupil and iris border.

The 30 percents normalization image furthermore is the main area where the analysis needs to be performed. For this thesis the analysis is carried out by using histogram function. The brightness of the image will be shown in bar chart of the histogram graph where the left side of $x$ axis will represent the by dark area showing low brightness, and the right side of $x$ axis will is represent by high level of brightness. The Histogram output than will be apply to OTSU threshold used to determine the threshold value of the image being analyzed. This OTSU threshold will give the average level of brightness in the eye image based on distribution of bar chart histogram from the output of histogram process. The OTSU graph will display and marked the threshold area for showing the threshold value.

Result from MATLAB later will display the string "Sodium ring is detected" or "no sodium ring is detected" depends on the eye image that be examined. For this experiment the boundary value of threshold in the examined eye image is set to 139 (this was decide after run 50 samples of the eye image), the average boundary of the illness or detected eye problem with sign of the presence cholesterol deposited. If the threshold value fallen below this value the eye image is considered as normal (no existing white ring or cholesterol), but if the threshold value rise up beyond this value (139), the subject or patient is detected a sign of the presence of cholesterol. In MATLAB window display will popup the message showing the result, base on value obtained in automated detecting cholesterol presence $(\mathrm{ADCP})$, written in MATLAB m-file programming. Results indicate the range of normal and abnormal eye, based on the threshold value obtain from ADCP. This will determine either someone have the symptom of the cholesterol presence or not. The result however is display in command window, but yet this program can be run and display using Graphic User Interface (GUI), where MATLAB has tool to perform it.

Normalization process is shown in Fig. 14, where in this process the image has been localized and transformed from polar to rectangular using gray image of normal eye. The transformation has to be cropped up to 30 percents from sclera/iris toward pupil because this is the area the Arcus Senilis normally exists.

Fig 15 shows the histogram, threshold values and the statement about the condition of the normal and Arcus Senilis iris, respectively. From the histogram and OTSU's method, the decidability or threshold value to distinguish between normal eyes and eyes with Arcus Lipids is found to be 139. This value is determined after testing 30 images of normal eyes. If the cluster mean value is less than this threshold, this means than the eye is normal eye and if it is above 139, then the eye can be detected as eye with Arcus Lipids. 

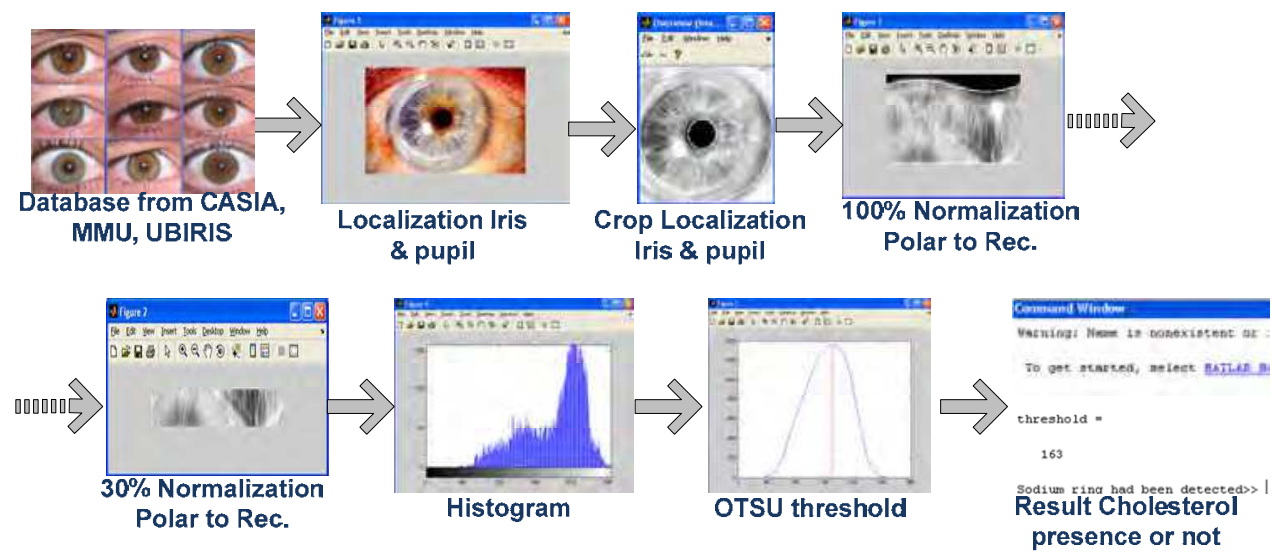

Fig. 13. Overall system for Cholesterol Detection.
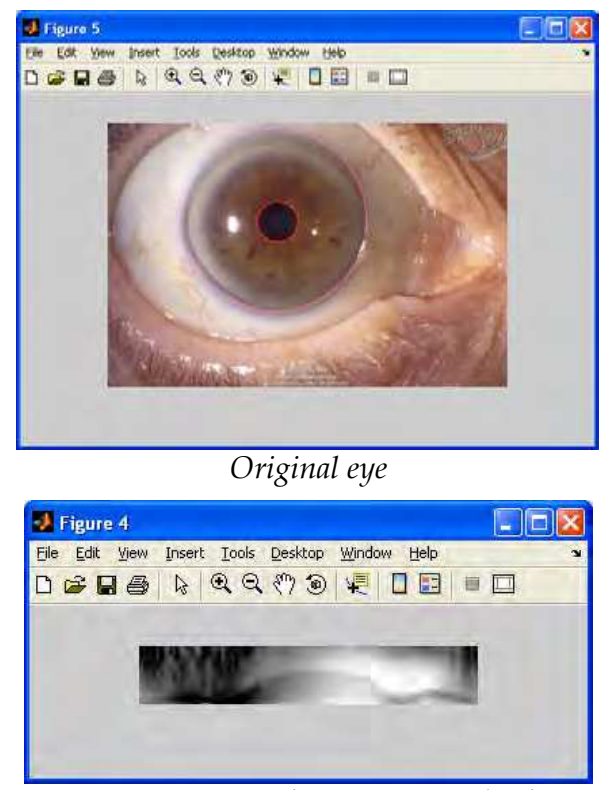

30 percents normalization image display

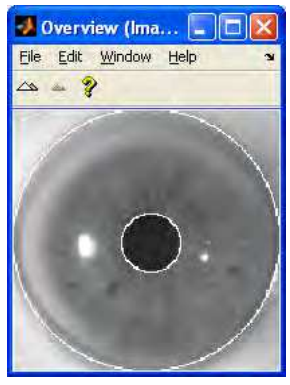

Segmentation and cropping process

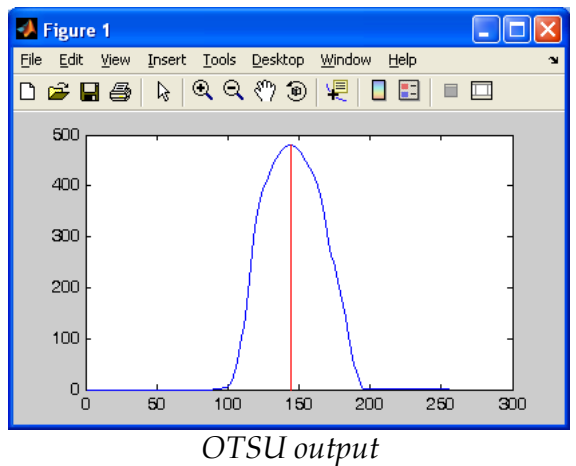

Fig. 14. Stages of localization with eye image 'Arcus1.bmp' from the medical web, (Clock wise from top left) original colour eye image localization, the iris and pupil detected correctly. (Top right) Gray eye image localization (Bottom left) 30 percents display from polar to rectangular of localization eye with enhancement. (Bottom right) OTSU threshold value for this eye is 144 . 


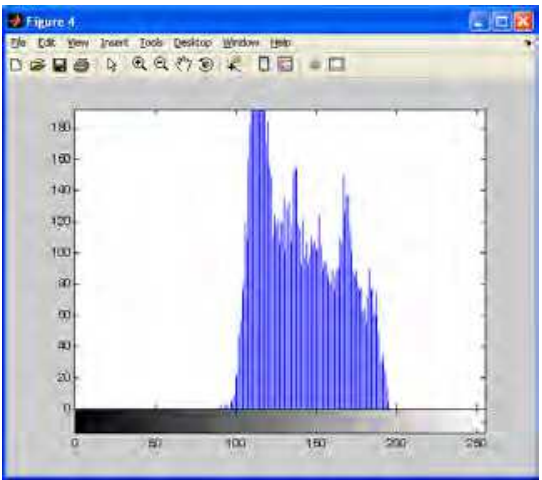

Histogram output

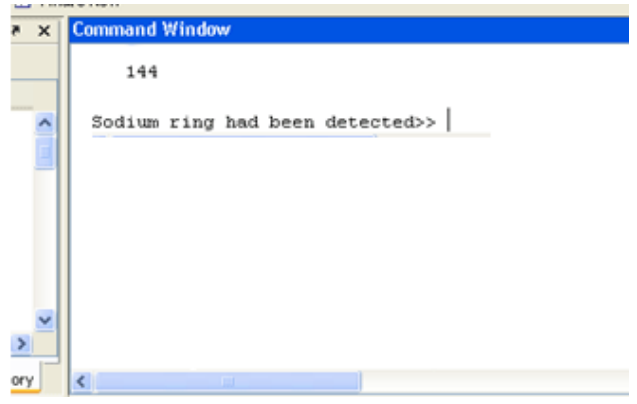

Final result

Fig. 15. Results from eye with "sodium ring' or Arcus senilis i.e. 'arcus1.bmp': histogram, threshold value and statement of iris condition.

Another result for cholesterol presence detection in normal eye shown as in Fig. 16 below, where the eye images in this figure shown variety of process involve from original eye until analysis to determine cholesterol presence.

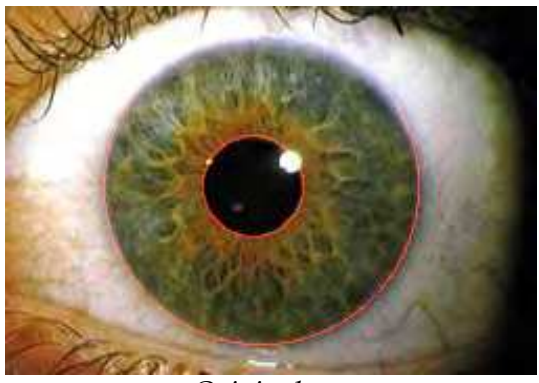

Original eye

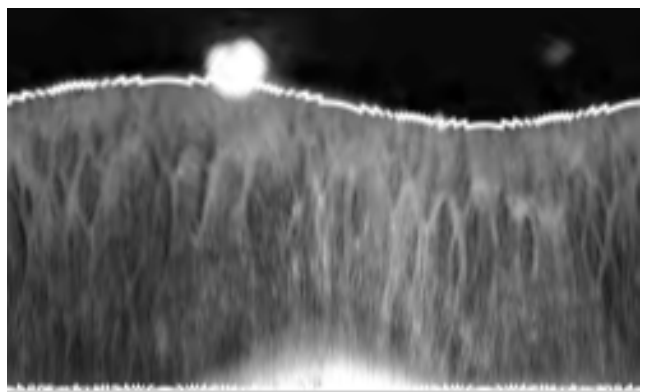

100 percents normalization image display

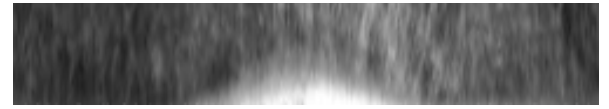

30 percents normalization image display

Fig. 16. Continued. 

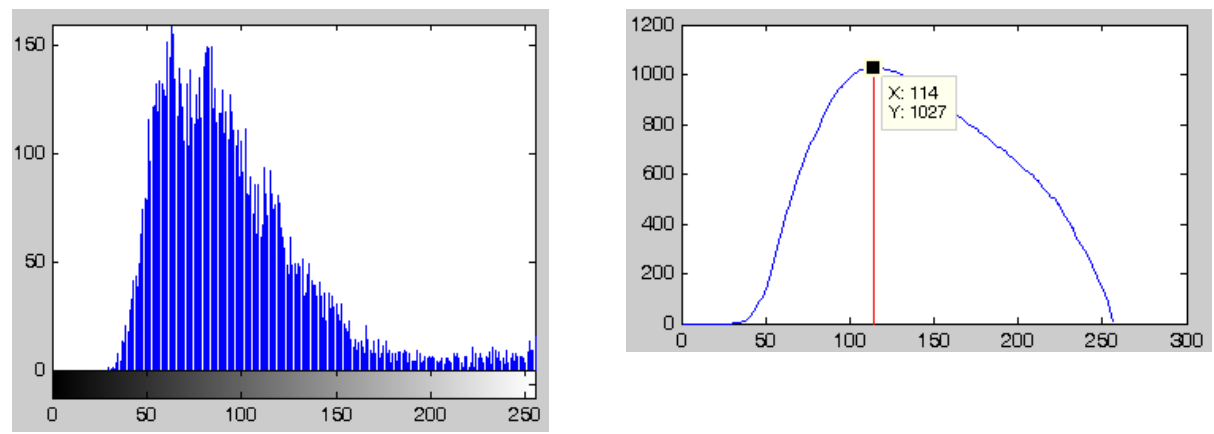

Histogram output

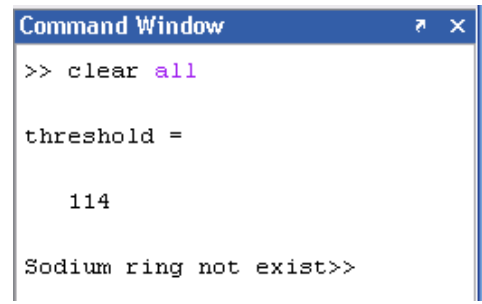

OTSU output

Final result

Fig. 16. Results from normal eye.

The output from this experiment shown in Fig. 16 indicate the eye image have no symptom of cholesterol presence this shown in command window result "Sodium ring not exist" and the threshold value give 114 which is below the set value (139). This meant the test eye image contain no symptom of cholesterol presence.

Another result from abnormal eye image is shown in Fig.17 below. The process follows the same method as describe in above procedure. For this image the threshold value determine is 148 which is higher than the set point 139 thus the result in command window display the message "Sodium ring had been detected", this indicate the eye encompass of cholesterol presence symptom.

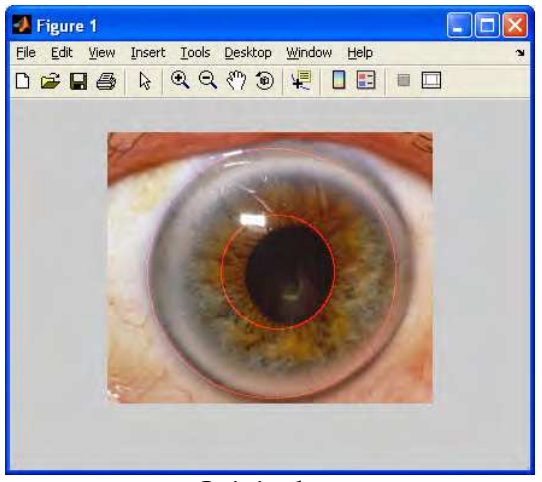

Original eye

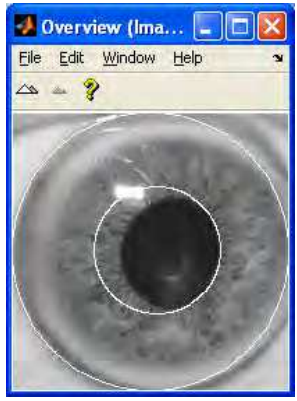

Segmentation and cropping process

Fig. 17. Continued. 

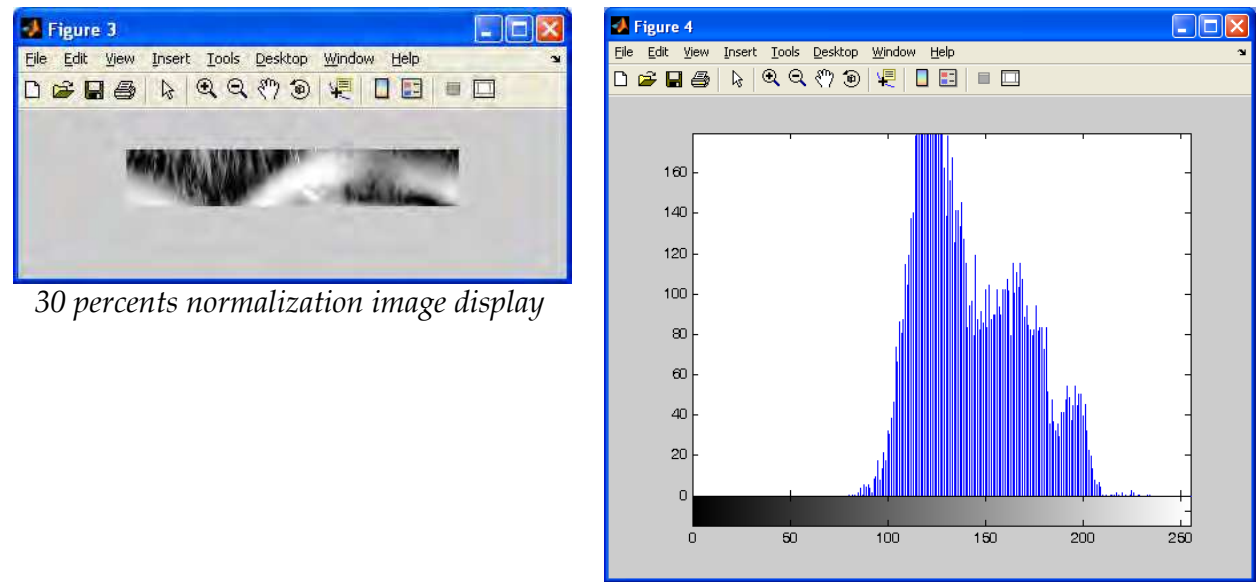

Histogram output
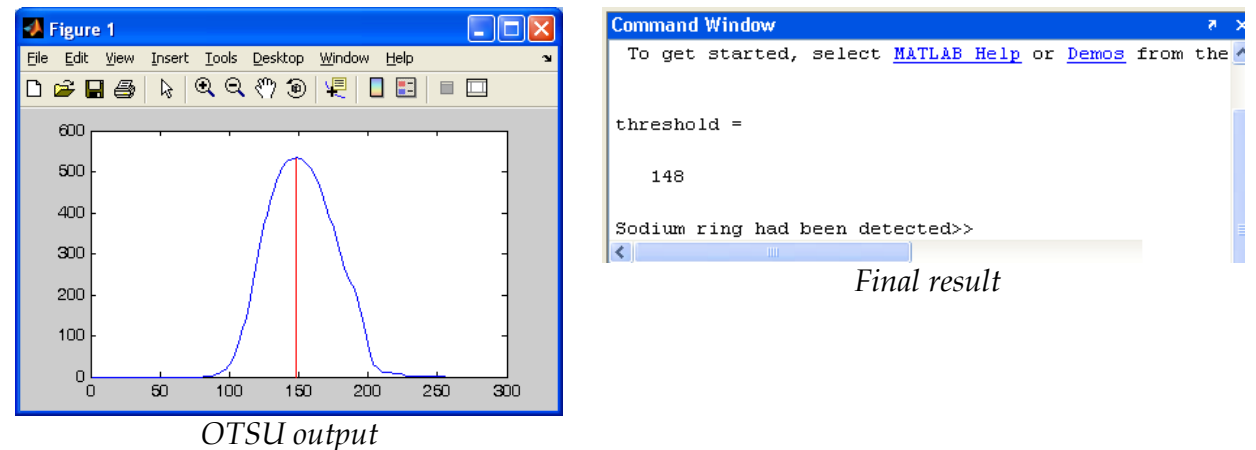

Final result

Fig. 17. Results from normal eye.

\section{Conclusion}

This work shows that there is a simple and non-intrusive method to detect cholesterol in body and iris recognition is not only mainly for biometric identification but it can also be used as a mean to detect cholesterol or maybe diagnose any diseases as iridology claimed it is supposed to be. However, this work is only preliminary work and experiments that are more extensive need to be run in future in order to know the real level of cholesterol in the body. This program had been executed on more than 50 samples of normal and abnormal eye images; it can be conclude that the threshold boundary of the normal and problem eye is about 139. This project had shown the entire process of detecting cholesterol presence using automated program (ADCP). However this programs written in $\mathrm{m}$-file and the result display in command window. The improvement can be done such as the using GUI for execute and displaying the result and using other method for determines the threshold of the normal or problem eye. Others application that can use this program is to determine the eye problem due to other type of eye diseases such as cataract, glaucoma, diabetic, tumour etcetera. 


\section{References}

[1] (N.Haq, M.D.Fox, 1991), N.Haq, M.D.Fox, "Preliminary results of IR spectroscopic characterization of cholesterol," Bioengineering Conference, 1991., Proceeding of the 1991 IEEE Seventeenth annual Northeast, pp.175-176, 4-5 Apr 1991.

[2] (FDA, 2004), U.S.Food and Drug Administration(FDA), “FDS Clears New Palm Test For Skin Cholesterol", FDA Talk Paper, June 24th, 2004, available at http://www.fda.gov/bbs/topics/ANSWERS/2002/ANS01154.html

[3] (L.Berggren, 1985), L.Berggren, "Iridology: a critical review" Acta Ophthalmol, 63,pp.18,1985 .

[4] (K.Hughes et. al., 1992), K.Hughes, K.C.Lun, S.P.Sothy, A.C. thai, W.P. Leong, P.B. Yeo, "Corneal arcus and cardiovascular risk factors in Asians in Singapore,"Int. Journal Epidemiol,vol.21, pp.473-147, 1992.

[5] (J. Daugman, 2004), J. Daugman. "How Iris Recognition Works," IEEE Transaction on Circuits and Systems for Video Technology, vol. 14, No.1, Jan 2004.

[6] (L. Masek, 2003) L. Masek, "Recognition of Human Iris Patterns for Biometric Identification", Dissertation, University of Western Australia, 2003.

[7] (CASIA, 2003), Chinese Academy ,Chinese Academy of Sciences - Institute of Automation. Database of 756 Greyscale Eye Images. http:/ / www.sinobiometrics.com Version 1.0, 2003.

[8] (MMU) Multimedia University -database contributes of 450 iris images http:// pesona.mmu.edu.my/ ccteo/

[9] UBIRIS Iris Image Databse, available at http://iris.di.ubi.pt/

[10] (UPOL) UPOL iris database, available at "http://phoenix.inf.upol.cz/iris/download/", update: 2003

[11] (NLM, 2010), National Library of Medicine - National Institutes of Health- world's largest medical "http:/ / www.nlm.nih.gov/" update: 2010

[12] (Theodore, RichardWildes 2002)Theodore A. Camus, RichardWildes, "Reliable and Fast Eye Finding in Close-up Images", This work was sponsored by the Defense Advanced Projects Agency.The views and conclusions contained in this document are those of the authors and should not be interpreted as representing the official policies, either expressly or implied, of the U.S. Defense Advanced Projects Agency, the U.S. Army Intelligence Center \& Fort Huachuca, Directorate of Contracting Office, or the U.S. Government.

[13] (Mediscan, 2000), Medical pictures and images -Mediscan clipart library Licensed medical pictures http://www.mediscan.co.uk/

[14] N.OTSU, A threshold selection method from gray-level histograms. IEEE Trans. on System, Man and Cybernetics, 9(1):62--66, 1979

[15] (Harold z. Pomerantz, 1962) Harold z. Pomerantz, M.D., Montreal, "The relationship Between Coronary Heart Disease and the Presence of Certain Physical Characteristics", Departments of Medicine and Cardiology, Reddy Memorial Hospital, Montreal, Canad. Med. Ass. J. Jan. 13, 1962, vol.86

[16] (Jae-Young Um et. al, 2005) Jae-Young Um,* Nyeon-Hyoung An,et. al, "Novel Approach of Molecular GeneticUnderstanding of Iridology: RelationshipBetween Iris Constitution and Angiotensin Converting Enzyme Gene Polymorphism", The American Journal of Chinese Medicine, Vol. 33, No. 3, 501-505, 2005 World 
Scientific Publishing Company Institute for Advanced Research in Asian Science and Medicine, 2005.

[17] (Frank L. Urbano, 2001) Frank L. Urbano, MD, "Ocular Signs of Hyperlipidemia", review of clinical signs,general internal medicine, Mount Laurel Primary Care Associates, Mount Laurel, NJ., Hospital Physician, November 2001

[18] Staessen, J.A., J.G. Wang, E. Brand, C. Barlassina, W.H. Birkenhager, S.M. Herrmann, R. Fagard, L. Tizzoni and G. Bianchi. The deletion/insertion polymorphism of the angiotensin converting enzyme gene and cardiovascular-renal risk. J. Hypertens. 19: 1349-1358, 2001.

[19] Ernst, E. Iridology: not useful and potentially harmful. Arch. Ophthalmol. 118: 120-121, 2000. 


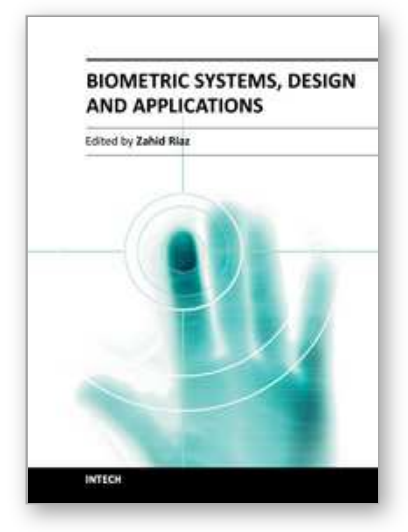

\author{
Biometric Systems, Design and Applications \\ Edited by Mr Zahid Riaz
}

ISBN 978-953-307-542-6

Hard cover, 262 pages

Publisher InTech

Published online 21, October, 2011

Published in print edition October, 2011

Biometric authentication has been widely used for access control and security systems over the past few years. The purpose of this book is to provide the readers with life cycle of different biometric authentication systems from their design and development to qualification and final application. The major systems discussed in this book include fingerprint identification, face recognition, iris segmentation and classification, signature verification and other miscellaneous systems which describe management policies of biometrics, reliability measures, pressure based typing and signature verification, bio-chemical systems and behavioral characteristics. In summary, this book provides the students and the researchers with different approaches to develop biometric authentication systems and at the same time includes state-of-the-art approaches in their design and development. The approaches have been thoroughly tested on standard databases and in real world applications.

\title{
How to reference
}

In order to correctly reference this scholarly work, feel free to copy and paste the following:

Ridza Azri Ramlee, Khairul Azha and Ranjit Singh Sarban Singh (2011). Detecting Cholesterol Presence with Iris Recognition Algorithm, Biometric Systems, Design and Applications, Mr Zahid Riaz (Ed.), ISBN: 978-953307-542-6, InTech, Available from: http://www.intechopen.com/books/biometric-systems-design-andapplications/detecting-cholesterol-presence-with-iris-recognition-algorithm

\section{INTECH}

open science | open minds

\author{
InTech Europe \\ University Campus STeP Ri \\ Slavka Krautzeka 83/A \\ 51000 Rijeka, Croatia \\ Phone: +385 (51) 770447 \\ Fax: +385 (51) 686166 \\ www.intechopen.com
}

\author{
InTech China \\ Unit 405, Office Block, Hotel Equatorial Shanghai \\ No.65, Yan An Road (West), Shanghai, 200040, China \\ 中国上海市延安西路65号上海国际贵都大饭店办公楼 405 单元 \\ Phone: +86-21-62489820 \\ Fax: $+86-21-62489821$
}


(C) 2011 The Author(s). Licensee IntechOpen. This is an open access article distributed under the terms of the Creative Commons Attribution 3.0 License, which permits unrestricted use, distribution, and reproduction in any medium, provided the original work is properly cited. 